\title{
A truncated Fourier-transform based approach for time-domain diffuse optical tomography
}

\author{
Meghdoot Mozumder, ${ }^{1, *}$ and Tanja Tarvainen ${ }^{2}$ \\ ${ }^{1}$ Department of Applied Physics, University of Eastern Finland, Kuopio, Finland \\ ${ }^{2}$ Department of Computer Science, University College London, London, United Kingdom \\ *meghdoot.mozumder@uef.fi
}

\begin{abstract}
We present a truncated Fourier series approximation for time-domain diffuse optical tomography. The approach estimated optical parameters with accuracies comparable to using the whole time-resolved data, using very low computational time and resources.

(c) 2020 The Author(s)
\end{abstract}

\section{Introduction}

Time-domain diffuse optical tomography (TD-DOT) uses pulsed near-infrared light for imaging spatially varying optical parameters in biological tissues. The image reconstruction problem of TD-DOT involves estimating spatially varying optical parameters, using time-resolved boundary measurements. Several approaches to solve the image reconstruction problem have been proposed. These include using the whole time-resolved measurement data and using integral-transform based moments of the time-resolved measurement data (e.g, Mellin and Laplace transforms) [1]. Use of such moments has led to a significant reduction of computation time, due to compression of the time-resolved measurement data. A comparison of estimation accuracies obtained using different choices of moments, using one in silico target, was presented in [1]. It was shown that using only one moment was inadequate to reconstruct both absorption and scattering parameters simultaneously. Also, there were inter-parameter crosstalks, such that the absorption and scattering estimates significantly affected each other. Alternatively, Gibson et al. [2] used difference imaging to reconstruct changes of optical parameters from one frequency of Fouriertransformed data. Here, we propose an approach to reconstruct optical parameters in TD-DOT using a truncated Fourier-series approximation [3].

\section{Theory}

\subsection{Truncated Fourier series approximation}

Let us consider the discretized TD-DOT observation model

$$
y_{t}=\Gamma_{t}\left(\mu_{a}, \mu_{s}^{\prime}\right)+e .
$$

Here, $y_{t}$ is the noisy time-resolved DOT data, $e$ is the additive noise, $\Gamma_{t}$ is a forward model of the time-domain light diffusion equation, and $\mu_{a}$ and $\mu_{s}^{\prime}$ are the spatially distributed absorption and reduced scattering coefficients. The truncated Fourier series approximation of the boundary measurement due to a (delta-function) source $\Gamma_{t}^{\delta}$ is given by,

$$
\Gamma_{t}^{\delta}=\sum_{k=1}^{\mathrm{N}_{\omega}} \Gamma\left(\omega_{k}\right) \exp \left(\mathrm{i} \omega_{k} t\right), \text { where } \Gamma\left(\omega_{k}\right)=\frac{1}{\mathrm{~T}} \int_{0}^{\mathrm{T}} \Gamma_{t}^{\delta} \exp \left(-\mathrm{i} \omega_{k} t\right)
$$

Here the temporal range of the function $\Gamma_{t}^{\delta}$ is $[0, T]$. For light sources with a finite temporal length, the output $\Gamma_{t}$ can be expressed as a convolution of the source pulse $\mathrm{Q}_{t}$ and exitance due to a delta source $\Gamma_{t}^{\delta}$. As such, taking a Fourier transform of measurements $\Gamma_{t}$ and using Convolution theorem, we can construct the normalized measurement data [3]

$$
\tilde{y}=\frac{y_{\mathrm{FT}}}{\mathrm{Q}_{\mathrm{FT}}}=\left(\begin{array}{l}
\operatorname{Re}\left(\Gamma\left(\omega_{k}\right)\right) \\
\operatorname{Im}\left(\Gamma\left(\omega_{k}\right)\right)
\end{array}\right)+\frac{e_{\mathrm{FT}}}{\mathrm{Q}_{\mathrm{FT}}}=\Gamma_{\omega}\left(\mu_{a}, \mu_{s}^{\prime}\right)+\tilde{e}
$$

where $y_{\mathrm{FT}}$ corresponds to Fourier-transformed data at frequencies $\omega_{k}$, $\mathrm{Q}_{\mathrm{FT}}$ is the Fourier-transform of the source pulse and $\tilde{e}=e_{\mathrm{FT}} / \mathrm{Q}_{\mathrm{FT}}$ is the normalized Fourier-transformed noise. The forward model $\Gamma_{\omega}$ can be modeled using the frequency-domain light diffusion equation. 

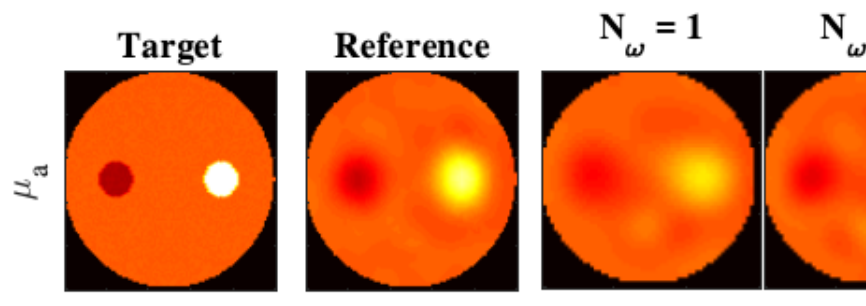

$\mathrm{N}_{\omega}=2$

$\mathrm{N}_{\omega}=\mathbf{3}$

$N_{\omega}=4$

$8 \%$
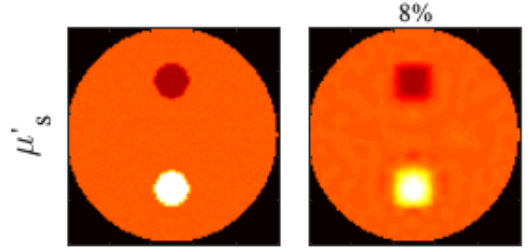

$2 \%$

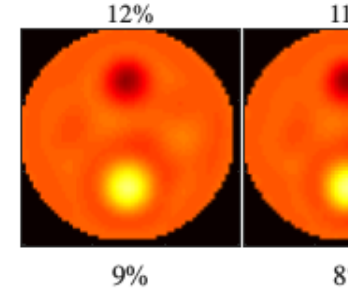

$9 \%$

$8 \%$

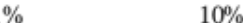

$10 \%-2=-10 \%$

$10 \%$

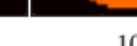

Fig. 1. Absorption (first row) and scattering (second row) distributions. First column: Simulated target. Second column: Reference estimates using whole time-domain data. Columns 3-6: Estimates using one to four Fourier frequencies $\left(N_{\omega}\right)$. Below each image is the relative estimation error.

\subsection{Bayesian approach to the inverse problem of TD-DOT}

The parameters $\left(\mu_{a}, \mu_{s}^{\prime}\right)$ were estimated using a Bayesian approach by minimizing

$$
\left(\hat{\mu}_{\mathrm{a}}, \hat{\mu}_{\mathrm{s}}^{\prime}\right)=\arg \min _{\mu_{a}, \mu_{s}^{\prime}}\left\{\left\|L_{\tilde{e}}\left(\tilde{y}-\Gamma_{\omega}\left(\mu_{a}, \mu_{s}^{\prime}\right)-\tilde{e}_{*}\right)\right\|^{2}+\left\|L_{\mu_{a}}\left(\mu_{a}-\mu_{a, *}\right)\right\|^{2}+\left\|L_{\mu_{s}^{\prime}}\left(\mu_{s}-\mu_{s, *}^{\prime}\right)\right\|^{2}\right\},
$$

using data at different number of frequencies, where $\tilde{e_{*}}$, is the noise mean and $L_{\tilde{e}}$ is the Cholesky of the inverse of noise covariance. Prior means $\left(\mu_{a, *}, \mu_{s, *}^{\prime}\right)$ and Cholesky matrices $\left(L_{\mu_{a}}, L_{\mu_{s}^{\prime}}\right)$ were specified using Gaussian Ornstein-Uhlenbeck prior. The reference estimates were calculated using the full time-resolved data $y_{t}$ as

$$
\left(\hat{\mu}_{\mathrm{a}}, \hat{\mu}_{\mathrm{s}}^{\prime}\right)=\arg \min _{\mu_{a}, \mu_{s}^{\prime}}\left\{\left\|L_{e}\left(y_{t}-\Gamma_{t}\left(\mu_{a}, \mu_{s}^{\prime}\right)-e_{*}\right)\right\|^{2}+\left\|L_{\mu_{a}}\left(\mu_{a}-\mu_{a, *}\right)\right\|^{2}+\left\|L_{\mu_{s}^{\prime}}\left(\mu_{s}-\mu_{s, *}^{\prime}\right)\right\|^{2}\right\},
$$

where $e_{*}$, is the noise mean and $L_{\tilde{e}}$ is the Cholesky of the inverse of noise covariance.

\section{Results}

In the numerical studies, the domain was a circle with radius $25 \mathrm{~mm}$, shown in the first column of Fig. 1 . The measurement setup consisted of 16 sources and 16 detectors, modeled as Gaussian surface patches with $2 \mathrm{~mm}$ width, located at equi-spaced angular intervals on the boundary. The reference estimate and estimates using four Fourier frequencies $\left(\mathrm{N}_{\omega}=1, \ldots, 4\right)$ is also displayed. The computation time was $18624 \mathrm{~s}$ for reference estimate; $21 \mathrm{~s}, 18 \mathrm{~s}, 45 \mathrm{~s}$ and $70 \mathrm{~s}$ for estimates with $\mathrm{N}_{\omega}=1, . .4$, i.e, the Fourier transformed estimates required very low computation time compared to the time-resolved data. The relative errors of these estimates are displayed below each image. The estimation errors did not change considerably after four frequencies and hence are not shown.

The results show that the proposed approach can recover reliable estimates of optical parameters, using only a few Fourier frequencies $(\sim 4)$, using significantly low computational resources. Based on the results, we suggest that the truncated Fourier series could provide an efficient modeling protocol for TD-DOT.

\section{References}

1. M. Schweiger, and S. R. Arridge, "Application of temporal filters to time resolved data in optical tomography,” Phys. Med. \& Biol. 44 (7), 1699 (1999).

2. A. P. Gibson, T. Austin, N. L. Everdell, M. Schweiger, S. R. Arridge, J. H. Meek, J. S. Wyatt, D. T. Delpy, and J. C. Hebden, "Three-dimensional whole-head optical tomography of passive motor evoked responses in the neonate," Neuroimage 30 (2), 521-528 (2006).

3. M. Mozumder, and T. Tarvainen, "Time-domain diffuse optical tomography utilizing truncated Fourier series approximation," JOSA A submitted (2019). 